\title{
Delayed Ileal Pouch Anal Anastomosis Has a Lower 30-Day Adverse Event Rate: Analysis From the National Surgical Quality Improvement Program
}

\author{
Bharati Kochar, MD, MSCR, ${ }^{*,+}$ Edward L. Barnes, MD, MPH, ${ }^{*,+}$ Anne F. Peery, MD, MSCR, ${ }^{+}$ \\ Katherine S. Cools, MD, ${ }^{\ddagger}$ Joseph Galanko, PhD, ${ }^{+}$Mark Koruda, MD, ${ }^{*, \neq}$ and Hans H. Herfarth, MD, PhD ${ }^{*,+}$
}

\begin{abstract}
Background: Ulcerative colitis (UC) patients requiring colectomy often have a staged ileal pouch anal anastomosis (IPAA). There are no prospective data comparing timing of pouch creation. We aimed to compare 30-day adverse event rates for pouch creation at the time of colectomy (PTC) with delayed pouch creation (DPC).

Methods: Using prospectively collected data from 2011-2015 through the National Surgical Quality Improvement Program, we conducted a cohort study including subjects aged $\geq 18$ years with a postoperative diagnosis of UC. We assessed 30-day postoperative rates of unplanned readmissions, reoperations, and major and minor adverse events (AEs), comparing the stage of the surgery where the pouch creation took place. Using a modified Poisson regression model, we estimated risk ratios (RRs) with $95 \%$ confidence intervals (CIs) adjusting for age, sex, race, body mass index, smoking status, diabetes, albumin, and comorbidities.
\end{abstract}

Results: Of 2390 IPAA procedures, 1571 were PTC and 819 were DPC. In the PTC group, 51\% were on chronic immunosuppression preoperatively, compared with $15 \%$ in the DPC group $(P<0.01)$. After controlling for confounders, patients who had DPC were significantly less likely to have unplanned reoperations (RR, 0.42; 95\% CI, 0.24-0.75), major AEs (RR, 0.72; 95\% CI, 0.52-0.99), and minor AEs (RR, 0.48; $95 \%$ CI, 0.32-0.73) than PTC.

Conclusions: Patients undergoing delayed pouch creation were at lower risk for unplanned reoperations and major and minor adverse events compared with patients undergoing pouch creation at the time of colectomy.

Key Words: inflammatory bowel disease, ulcerative colitis, surgery

\section{INTRODUCTION}

The prevalence of ulcerative colitis (UC) in the United States is rising; currently nearly 900,000 Americans are affected. ${ }^{1,2}$ Over the course of the disease, approximately $30 \%$ of UC patients require surgical management. ${ }^{3}$ Since its first

Received for publications October 30, 2017; Editorial Decision January 2, 2018

From the ${ }^{*}$ Multidisciplinary Center for Inflammatory Bowel Disease, ${ }^{\dagger}$ Center for Gastrointestinal Biology and Disease, and ${ }^{*}$ Department of Surgery, University of North Carolina, Chapel Hill, North Carolina

Conflicts of interest: Hans H. Herfarth: consultant for Merck, Celltrion, Lycera. All other authors declare no conflicts of interest.

Supported by: This research was supported by grants from the National Institutes of Health (T32DK07634 [B.K.], K23DK113225 [A.F.P.], R25CA116339 [K.S.C.], P30DK349897 [J.G.] and 5U01DK092239 [H.H.H.]) and the Crohn's and Colitis Foundation (H.H.H.).

Disclosure: The American College of Surgeons National Surgical Quality Improvement Program (ACS NSQIP) and the hospitals participating in the ACS NSQIP are the source of the data used herein; they have not verified and are not responsible for the statistical validity of the data analysis or the conclusions derived by the authors.

Address correspondence to: Hans $\mathrm{H}$. Herfarth, MD, PhD, Division of Gastroenterology and Hepatology, University of North Carolina, Bioinformatics Building, CB\#7080, Chapel Hill, NC, 27599 (hherf@med.unc.edu).

(C) 2018 Crohn's \& Colitis Foundation. Published by Oxford University Press. All rights reserved. For permissions, please e-mail: journals.permissions@oup.com.

doi: 10.1093/ibd/izy082 Published online 25 April 2018 description in 1978, the total proctocolectomy with ileal pouch anal anastomosis (IPAA) creation has become the surgical procedure of choice for patients with medically refractory ulcerative colitis. ${ }^{3-5}$ The increasing prevalence of UC, along with the decreasing use of total proctocolectomy with end ileostomy, has resulted in an increase in the number of IPAA procedures. ${ }^{2,6,7}$

IPAA is often a staged procedure. The colectomy and creation of the pouch can be done safely as a single procedure in highly selected patients. ${ }^{3,8}$ However, it is more often done as a multistaged procedure, especially in patients with active ulcerative colitis. ${ }^{8,9}$ The traditional 2-stage procedure is a total proctocolectomy with ileal pouch creation and anastomosis along with a diverting loop ileostomy in the first stage and an ileostomy take down as the second stage. The traditional 3-stage procedure is a total colectomy with an end ileostomy in the first stage, completion proctectomy with ileal pouch creation, and anal anastomosis along with a diverting loop ileostomy in the second stage and ileostomy take down as the third stage. Some surgeons perform a variant 2-stage procedure with pouch creation in the second operation. ${ }^{10,11}$ Despite the frequent use of these multistaged surgical approaches, there are very few studies comparing them. The existing literature comparing early pouch creation (1- or 2-stage procedures) with delayed pouch creation (modified 2-stage or 3-stage procedures) is sparse, from single centers, and includes small numbers of patients. ${ }^{8,9,12}$ 
Given these knowledge gaps in the literature, there is a strong need for evaluation of adverse event rates after IPAA creation with prospectively collected data across multiple sites around the United States. Furthermore, it would be beneficial to compare outcomes between the multistaged procedures. We hypothesize that immunosuppression at the time of pouch creation is most likely to result in worse postoperative outcomes. To test this hypothesis, we analyzed data from the American College of Surgeons' (ACS) National Surgical Quality Improvement Program (NSQIP).

\section{METHODS}

We conducted a cohort study using data collected prospectively as part of the ACS NSQIP. Developed in 1994, NSQIP is a nationally validated program to measure and improve surgical outcomes. Participation in NSQIP is voluntary. NSQIP included 315 US hospitals in 2011, 374 hospitals in 2012, 435 hospitals in 2013, and 517 hospitals in 2014 and 2015. The development of NSQIP has been described previously. ${ }^{13,14}$ In brief, data are collected by trained surgical reviewers for more than 150 well-defined variables. These variables span preoperative risk factors, intra-operative variables, and 30-day postoperative outcomes. Routine inter-rater reliability audits are conducted to ensure high-quality data. Each surgery is collected in a discrete and de-identified manner, not linked to any other surgeries. The Institutional Review Board of the University of North Carolina at Chapel Hill determined that this study was exempt from continuing review due to the de-identified nature of the data.

\section{Study Population}

We included all patients in NSQIP between 2011 and 2015, 18 years or older, who had a postoperative diagnosis for ulcerative colitis (International Classification of Diseases, Ninth Revision [ICD-9] code 556 or ICD-10 K51) and a Current Procedural Terminology (CPT) code for ileal pouch anal anastomosis $(44158,44211$, and 45113). In this analysis, we compared the stage of the surgery where the pouch creation took place (Fig. 1). The traditional 2-stage IPAA where the pouch is created with the colectomy as a first surgery was designated pouch creation at the time of colectomy (PTC). The traditional 3-stage surgery where the pouch is created in a second surgery after the colectomy was termed delayed pouch creation. In some institutions, delayed pouch creation is done as a modified 2-stage operation (Fig. 1), which was analyzed with the delayed pouch creation group. ${ }^{10} \mathrm{CPT}$ codes 44158 and 44211 were used to designate PTC, where the pouch is created with the first operation. CPT code 45113 was used to designate delayed pouch creation.

In a subanalysis, we compared patients undergoing a colectomy without pouch creation (the first stage of the traditional 3 -stage IPAA surgery) to PTC. We used CPT codes 44155 , 44156, 44210, and 44212 to find total abdominal colectomies (TAC) with ileostomy creation.

We assessed the following patient characteristics before surgery: age, sex, race, body mass index, current smoking status, diabetes, and preoperative albumin. As NSQIP is not an administrative claims database, the American Society of Anesthesiologists (ASA) class is the marker for comorbidities. We also assessed preoperative immunosuppression use. Preoperative immunosuppression is 1 variable in this database; it is defined as the regular use of oral or parenteral corticosteroid medications or immunosuppressant medications within 30 days of the operative procedure or at the time the patient was being considered as a candidate for surgery. Specific classes or doses of immunosuppressant medications are not reported in the database.

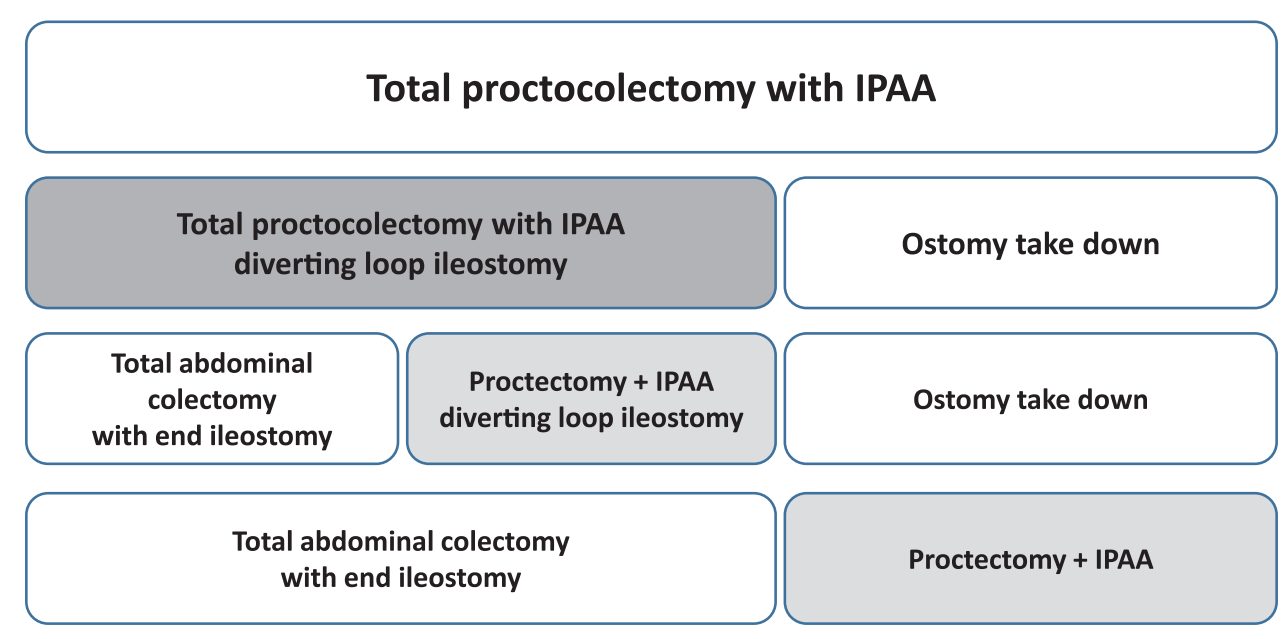

FIGURE 1. Stages of ileal pouch anal anastomosis procedures compared. Legend: Each box represents a surgery. The first line depicts the 1-stage IPAA procedure. The second line depicts the traditional 2-stage IPAA procedure. The third line depicts the traditional 3-stage IPAA procedure. The fourth line depicts the modified 2-stage IPAA procedure. The shaded boxes represent the surgery in which the pouch creation takes place. In the analyses presented, we compared outcomes after pouch creation at the time of colectomy (darker shade) with delayed pouch creation (lighter shade). 
Adverse events within 30 days of the pouch creation surgery were reported. Major adverse events and minor adverse events were defined by the Clavien-Dindo classification system. ${ }^{15}$ Major adverse events included organ space surgical site infection (SSI), sepsis, septic shock, acute renal failure, stroke/ cerebrovascular accident, myocardial infarction, cardiac arrest requiring cardiopulmonary resuscitation, time on ventilator greater than 48 hours, hospitalization greater than 30 days, or death. Minor adverse events included wound disruption, deep incisional SSI, superficial SSI, urinary tract infection, pneumonia, deep venous thrombosis/thrombophlebitis requiring therapy, and pulmonary embolus. Unplanned readmission and unplanned reoperation are reported separately.

\section{Statistical Analysis}

The rates of major and minor adverse events were calculated and stratified by timing of pouch creation. Modified Poisson regression with robust error variances was used to estimate risk ratios (RRs) and 95\% confidence intervals for unplanned readmission, unplanned reoperation, and major and minor adverse events by time of pouch creation. Multivariable models were created, which generated adjusted risk estimates.
The final model included age, sex, race, body mass index (BMI), smoking status, diabetes, ASA class, and preoperative albumin. Each of these potential confounders was chosen a priori based on clinical judgement. We did not control for preoperative immunosuppression as we hypothesized that it is on the casual pathway for the outcomes of interest. The same analyses were repeated for the subanalysis comparing TAC with ileostomy with PTC. $P$ values of less than 0.05 were considered statistically significant. The analyses were performed using SAS 9.4 (SAS Institute, Cary, NC, USA).

\section{RESULTS}

There were 2390 subjects who underwent an IPAA procedure; 1571 underwent PTC whereas 819 underwent delayed pouch creation (Table 1). The median age in the overall cohort was 39 years (interquartile range [IQR], 29-51 years). The cohort was $42 \%$ female and $86 \%$ white.

The cohort that had PTC did not have any clinically meaningful differences in their BMI, smoking status, history of diabetes mellitus, preoperative laboratory values, and ASA class compared with the cohort that had delayed pouch creation. Patients undergoing PTC had a median length of stay 1 day greater than those

TABLE 1: Characteristics of the Study Population Undergoing Ileal-Pouch Anal Anastomosis Creation

\begin{tabular}{|c|c|c|c|}
\hline Characteristic & $\begin{array}{l}\text { Pouch creation } \\
\text { at the time } \\
\text { of colectomy } \\
(\mathrm{n}=1571)\end{array}$ & Delayed Pouch $(\mathrm{n}=819)$ & $P$ \\
\hline Median age (IQR), y & $40(30-52)$ & $37(27-50)$ & $<0.01$ \\
\hline$\%$ female & 41 & 42 & 0.66 \\
\hline$\%$ white & 87 & 85 & 0.23 \\
\hline$\%$ BMI category & & & 0.48 \\
\hline$<18.5$ & 4 & 4 & \\
\hline $18.5-25$ & 41 & 38 & \\
\hline $26-30$ & 34 & 37 & \\
\hline$>30$ & 21 & 21 & \\
\hline$\%$ current smoker & 6 & 7 & 0.18 \\
\hline$\%$ diabetic & 6 & 5 & 0.32 \\
\hline$\%$ chronic immunosuppressant $^{\mathrm{a}}$ & 51 & 15 & $<0.01$ \\
\hline Median preoperative albumin (IQR), g & $3.9(3.5-4.2)$ & $4.2(3.8-4.5)$ & $<0.01$ \\
\hline Median preoperative white blood cell count (IQR), $10 * 9 /$ Liter & $7.8(6.1-10)$ & $7.0(5.9-8.4)$ & $<0.01$ \\
\hline Median preoperative hematocrit (IQR), \% & $39(35-42)$ & $40(37-43)$ & $<0.01$ \\
\hline$\%$ ASA class & & & 0.78 \\
\hline 1 & 2 & 3 & \\
\hline 2 & 69 & 67 & \\
\hline 3 & 29 & 30 & \\
\hline Median length of stay (IQR), d & $6(4-9)$ & $5(4-8)$ & $<0.01$ \\
\hline Median operative time (IQR), min & $276(212-353)$ & $213(168-278)$ & $<0.01$ \\
\hline
\end{tabular}

${ }^{a}$ Chronic immunosuppressant includes steroids, and chronic is defined as $>30$ days before the procedure or at the time of evaluation for the procedure. $\mathrm{IQR}=$ inter-quartile range. 
undergoing delayed pouch creation ( 6 days vs 5 days, $P<0.01$ ). In comparison with patients with delayed pouch creation, patients undergoing PTC were significantly more likely to be treated with a chronic immunosuppressant ( $51 \%$ vs $15 \%, P<0.01$ ).

The overall risk of 30-day mortality after an IPAA creation was $0.1 \%$, with no deaths in the delayed pouch creation group. The risk of 1 or more major adverse events postoperatively was significantly higher in the PTC group compared with the delayed pouch creation group $(12.4 \%$ vs $8.7 \%, P<0.01)$ (Fig. 2). The risk of 1 or more minor adverse events postoperatively was also higher in the PTC group compared with the delayed pouch creation group $(11.8 \%$ vs $6.1 \%, P<0.01)$. The risk of unplanned readmission after an IPAA procedure was similar in both the PTC and delayed pouch creation groups ( $23.3 \%$ vs $21.3 \%, P=0.26$ ). However, the risk of unplanned reoperations was higher in the PTC group compared with the delayed pouch creation group $(7.7 \%$ vs $3.8 \%, P<0.01)$. The delayed pouch creation group had a significantly lower rate of organ space surgical site infections, urinary tract infections, sepsis, and thrombotic events compared with the PTC group (Table 2).

After controlling for confounders (age, sex, race, BMI, smoking status, diabetes mellitus, preoperative albumin, and ASA class) subjects who had delayed pouch creation had a significantly decreased risk of unplanned reoperation (RR, 0.42 ; $95 \%$ confidence interval $[\mathrm{CI}], 0.24-0.74)$ compared with those who had PTC (Table 3). Those who underwent delayed pouch creation had a significantly decreased risk of major adverse events (RR, 0.72 ; $95 \%$ CI, 0.52-0.99) compared with those who had PTC. Sepsis and organ space surgical site infections were the primary contributors to this difference in major adverse events between those who underwent delayed pouch creation and PTC.
Subjects who had delayed pouch creation were also at a significantly lower risk for minor adverse events within 30 days after surgery compared with those who had PTC (RR, $0.48 ; 95 \%$ CI, $0.32-0.73$ ). Urinary tract infections and thrombotic events (both deep vein thrombosis and pulmonary embolism) were the predominant contributors to this difference in minor adverse events between those who underwent delayed pouch creation and PTC.

Reasons for readmission were available for $73 \%$ (392 subjects) of those who had an unplanned readmission (Fig. 3). Among these subjects, infections were the leading reasons for readmissions $(33 \%)$, fluid, electrolytes, and nutrition issues accounted for $18 \%$ of readmissions, and ileus or obstruction accounted for $15 \%$ of readmissions.

To evaluate if the first surgical procedure for patients with UC represents a higher-risk for adverse events in general, in a subanalysis, we compared those who had a TAC with ileostomy with those who had PTC. In the NSQIP database, there were 2460 subjects undergoing a TAC with ileostomy. This population was $45 \%$ female and $85 \%$ white with a median age of 46 years. Compared with those who had a PTC, those who had a TAC with ileostomy were more likely to be diabetics $(8 \%$ vs $6 \%, P=0.02)$ and to have been on steroids or immunosuppression 30 days before surgery ( $69 \%$ vs $51 \%, P<0.01)$. They were also more likely to be classified as ASA III or greater $(47 \%$ vs $29 \%, P<0.01$ ) and to have a lower preoperative albumin (3.3 grams vs 3.8 grams, $P<0.01$ ). However, after adjusting for confounders, those undergoing a TAC with ileostomy were significantly less likely to have unplanned readmissions (RR, $0.69 ; 95 \%$ CI, $0.58-0.81$ ), major complications ( $\mathrm{RR}, 0.68 ; 95 \%$ CI, 0.54-0.84), and minor complications (RR, $0.79 ; 95 \% \mathrm{CI}$, 0.63-0.98) compared with those who had PTC.

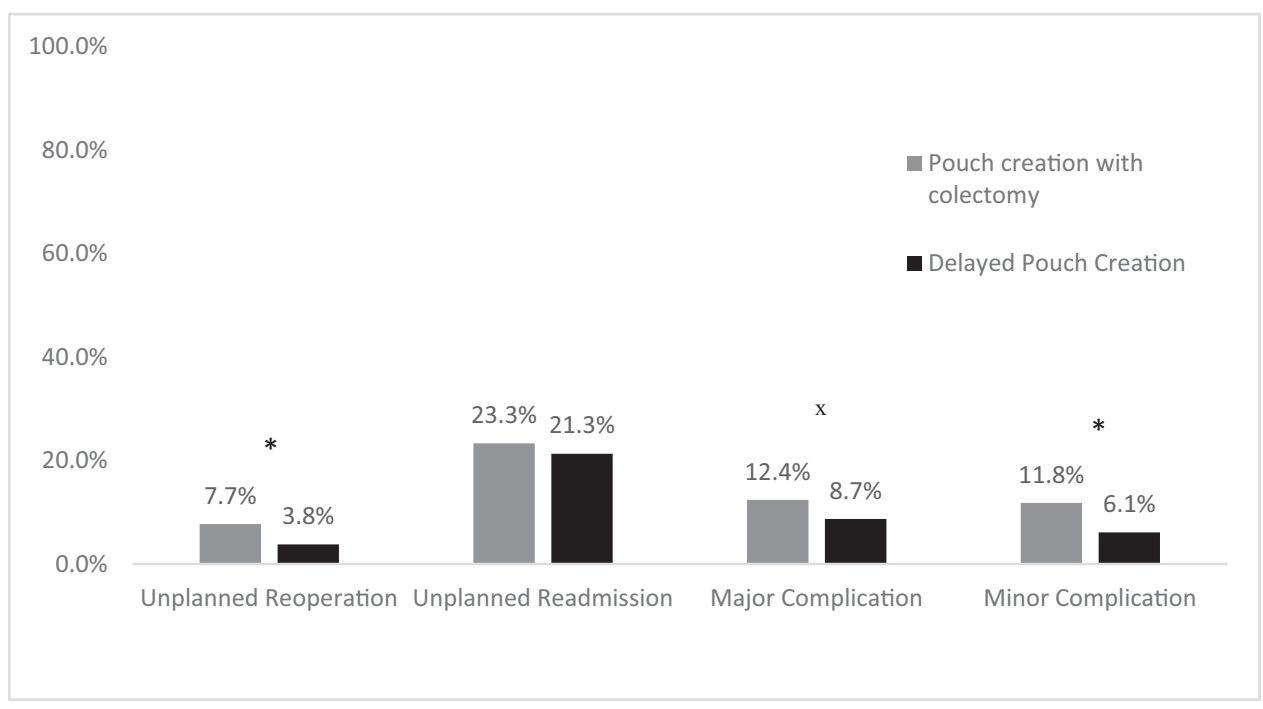

FIGURE 2. Rates of 30-day adverse events after ileal pouch anal anastomosis procedures. Legend: $* P<0.001 ; \times P=0.008$. A major adverse event is defined as an organ space SSI, sepsis, septic shock, acute renal failure, stroke/cerebrovascular accident, myocardial infarction, cardiac arrest requiring cardiopulmonary resuscitation, time on ventilator greater than 48 hours, hospitalization greater than 30 days, or death. A minor adverse event is defined as a wound disruption, deep incisional SSI, urinary tract infection, pneumonia, deep venous thrombosis/thrombophlebitis requiring therapy, and pulmonary embolus. 
TABLE 2: Adverse Events after lleal Pouch Anal Anastomosis Creation

\begin{tabular}{lllr}
\hline Adverse Event & $\begin{array}{c}\text { PTC } \\
(\mathrm{n}=1571)\end{array}$ & $\begin{array}{c}\text { Delayed } \\
\text { Pouch (n }=819)\end{array}$ & \multicolumn{1}{c}{$P$} \\
\hline $\begin{array}{l}\text { Organ space surgical } \\
\quad \text { site infection }\end{array}$ & 9 & 6 & $<0.01$ \\
$\begin{array}{l}\text { Sepsis } \\
\text { Septic shock }\end{array}$ & 6.6 & 4.3 & 0.02 \\
Acute renal failure & 0.5 & 0.6 & 0.75 \\
Cerebrovascular event & 1.2 & 0.7 & 0.34 \\
Myocardial infarction & 0 & 0 & \\
Cardiac arrest & 0.1 & 0.1 & 0.97 \\
Failure to wean & 0.2 & 0 & 0.21 \\
$\quad$ from the ventilator & 0.4 & 0.2 & 0.58 \\
Length of stay $>30 \mathrm{~d}$ & 0.6 & & 0.2 \\
Superficial surgical & 4.5 & 7.1 & $<0.01$ \\
$\quad$ site infection & & & \\
Deep incisional surgical & 2 & 1.2 & 0.18 \\
$\quad$ site infection & & & \\
Wound disruption & 1.2 & 0.6 & 0.20 \\
Urinary tract infection & 4.7 & 2.3 & $<0.01$ \\
Pneumonia & 0.6 & 0.7 & 0.64 \\
Deep vein thrombosis & 4 & 1.8 & $<0.01$ \\
Pulmonary embolus & 1.2 & 0.2 & 0.02 \\
\hline
\end{tabular}

All numbers are percentages unless otherwise specified.

PTC $=$ pouch creation at the time of colectomy.

\section{DISCUSSION}

This is the first prospective assessment of morbidity after IPAA creation in UC patients stratified by timing of pouch creation from a national database. We determined adverse event rates for UC patients after the pouch creation surgery in an IPAA procedure. Mortality was exceedingly low $(0.1 \%)$ in this cohort. Compared with patients who had a pouch created at the time of colectomy (PTC), patients who had delayed pouch creation were at a significantly lower risk for unplanned reoperations, major adverse events, such as organ space surgical site infections, and minor adverse events, such as thrombotic events, within 30 days after surgery. Infections, fluid, electrolyte, and nutrition problems account for half the reasons for readmissions after IPAA procedures.

Currently in the United States, outcomes after total proctocolectomy with IPAA creation are mostly described from the experience of 2 large quaternary care centers. ${ }^{8}{ }^{16-23}$ Metaanalyses are compilations of experiences from smaller centers, which are largely retrospective. ${ }^{9,24,25}$

One large study reported a $21 \%$ overall post-IPAA complication rate. ${ }^{26}$ This health system report of 30-day readmission after IPAA surgery noted an overall readmission rate of $23 \%$, double that of other colorectal procedures in their cohort. This study also reported that the majority of IPAAs were done
TABLE 3: Adjusted Risk of 30-Day Adverse Events Following Ileal Pouch Anal Anastomosis Creation

Adverse Event

Delayed Pouch (ref: PTC)

Unplanned reoperation

$0.42(0.24-0.75)$

Unplanned readmission

$0.95(0.77-1.16)$

Major adverse event ${ }^{\mathrm{a}}$

$0.72(0.52-0.99)$

Minor adverse event ${ }^{\mathrm{b}}$

$0.48(0.32-0.73)$

Risk ratios after controlling for age, sex, race, BMI, smoking status, diabetes, preoperative albumin, and American Society of Anesthesiology class.

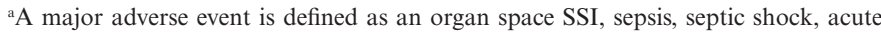
renal failure, stroke/cerebrovascular accident, myocardial infarction, cardiac arrest requiring cardiopulmonary resuscitation, time on ventilator greater than 48 hours, hospitalization greater than 30 days, or death.

${ }^{\mathrm{b}} \mathrm{A}$ minor adverse event is defined as a wound disruption, deep incisional SSI, superficial SSI, urinary tract infection, pneumonia, deep venous thrombosis/thrombophlebitis requiring therapy, and pulmonary embolus.

PTC $=$ pouch creation at the time of colectomy.

in low-volume centers that performed fewer than 15 IPAA procedures a year. They showed that readmission rates were related to the center's IPAA volume, with lower-volume centers having significantly higher rates of readmission. ${ }^{27}$ This study was not able to specify adverse event rates by timing of pouch creation, as we did.

There are only a few studies assessing timing of pouch creation. There is no consensus on whether early or delayed pouch creation is more optimal for UC patients. One hypothesis is that increasing the number of surgeries decreases quality of life. However, a small study concluded that there was no difference in quality of life between those who underwent a 2-stage IPAA procedure and a 3 -stage IPAA procedure. ${ }^{28}$ Another thought is that preoperatively sicker and immunosuppressed patients more often undergo procedures with delayed pouch creation. ${ }^{29}$ Although this may be the norm at high-volume centers, our data from a national sample indicate that this is not standard. One single-center study suggested that preoperative steroids and anti-tumor necrosis factor agents do not seem to justify the decision to avoid a 2-stage approach as outcomes may be more influenced by a surgeon's experience. ${ }^{12}$ However, our data show that patients who had a delayed pouch creation had significantly fewer adverse events 30 days postoperation, which is most likely explained by the significantly lower rate of chronic immunosuppressive therapy before pouch creation. Our data are concordant with a recent single-center study comparing the traditional 2-stage IPAA with a modified 2-stage IPAA with delayed pouch creation, which concluded that those who had delayed pouch creation had a significantly lower rate of postoperative anastomotic leak. ${ }^{30}$

In a subanalysis comparing the first surgeries (TAC with end ileostomy vs PTC), we demonstrate that despite being sicker at the time of initial surgery with higher rates of immunosuppression, patients undergoing TAC with end ileostomy 


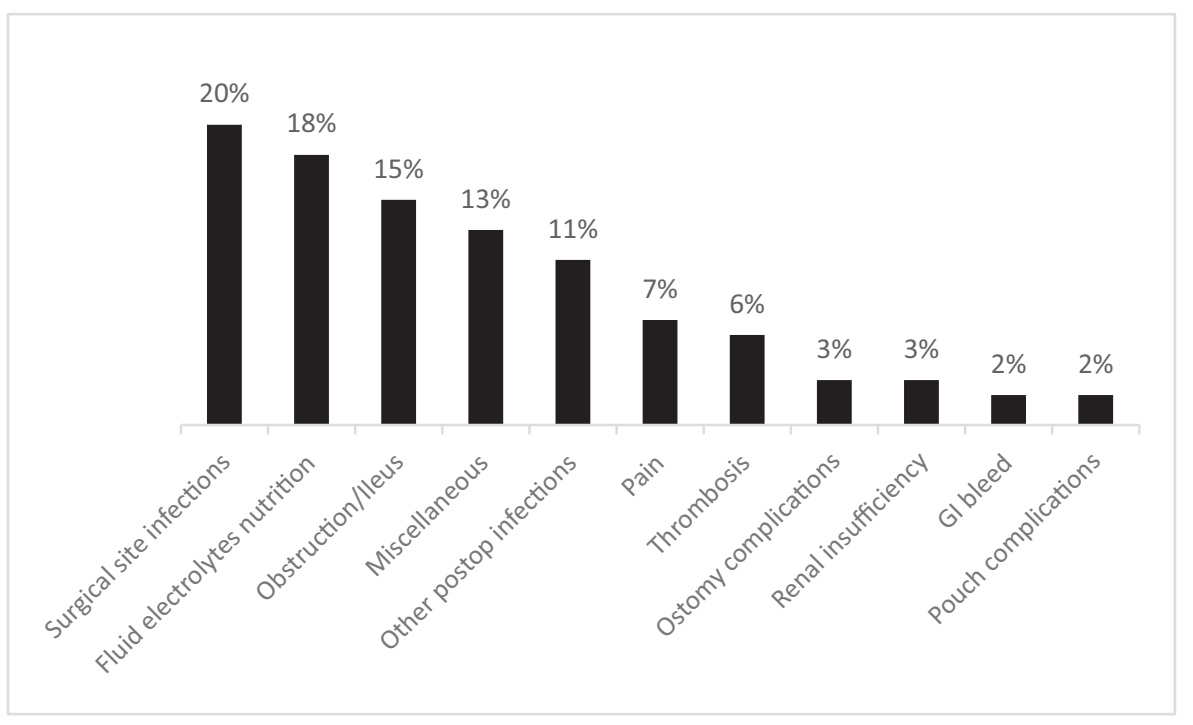

FIGURE 3. Reasons for readmission after ileal pouch anal anastomosis creation. Abbreviation: Gl, gastrointestinal.

were significantly less likely to experience a postoperative complication such as unplanned readmissions and major and minor complications. This supports the argument that pouch creation is an aspect of the surgery that may be influencing postoperative outcomes. Therefore, pouch creation should be delayed, especially if a patient is on preoperative immunosuppressive therapy.

Data regarding preoperative immunosuppression for pouch surgery are conflicting. An older study in administrative claims data found that prescriptions for immunosuppression before a colectomy did not increase the risk for postoperative complications. ${ }^{31}$ A more recent study in administrative claims data demonstrated that UC patients undergoing a colectomy with IPAA on an anti-tumor necrosis factor (anti-TNF) agent had an increased rate of postoperative adverse events compared with patients not on an anti-TNF agent 90 days preoperation. ${ }^{32}$ In our cohort, chronic immunosuppressant use before surgery was significantly higher in the PTC group compared with the delayed pouch creation group. This is likely explained by the fact that those who had a pouch created with the colectomy are often on medical therapy preceding the colectomy, whereas those who had delayed pouch creation often have immunosuppression held after the colectomy.

In a recent claims study of UC patients undergoing colectomies, UC patients on anti-TNF agents undergoing a subtotal colectomy or colectomy with ileostomy did not have the same increased risk of postoperative adverse events as those undergoing an IPAA, which is corroborated by our findings. ${ }^{32}$ This suggests that pouch creation is a unique aspect of colectomies for UC patients that may be more influenced by recent immunosuppressive therapy. Medical gastroenterologists are often asked to provide opinions regarding preoperative optimization. Therefore, understanding the role of preoperative immunosuppression and the surgery that will be performed will be informative for the gastroenterologist and their patient.

There are many strengths to our study. Including nearly 5000 patients undergoing surgery for $\mathrm{UC}$ from more than 500 hospitals across the United States over 5 years, this analysis is one of the largest studies comparing multistaged IPAA procedures in UC patients. This is also one of the few studies of IPAA procedures where the data were collected prospectively. The ACS NSQIP collects data in a standardized manner with a systems of checks in place to assure reliability. Additionally, the ACS NSQIP data have been used before to report postoperative outcomes for inflammatory bowel disease patients. ${ }^{33-37}$ Our study has limitations as well. The ACS NSQIP is not representative of all hospitals across the United States. Participation is voluntary, and a disproportionate number of academic hospitals participate in the program. Additionally, as it is a quality improvement program, participation in itself may improve outcomes; therefore, we may have underestimated postoperative adverse events. Given the nature of the NSQIP database, we cannot link staged surgeries and compare overall outcomes, we can only compare 1 stage of surgery to another. However, as pouch creation is hypothesized to be the sensitive part of this surgical process, comparing the stages where the pouch creation takes place is an appropriate surrogate.

Our subanalysis may also be flawed as not all those who undergo a TAC with ileostomy proceed to have pouch creation for various clinical reasons, including length of surgery and pelvic dissection. However, this comparison was meant to provide an estimate of risk for the first stage of the traditional 3-stage IPAA and illustrate the burden of immunosuppression associated with this stage of surgery. As the data were not collected for the purposes of evaluation of inflammatory bowel disease patients, we could not control for preoperative 
disease activity and detail the type of chronic immunosuppression used, which is needed to draw a more definitive conclusion. Additionally, numerous studies note that center experience and surgeon volume are predictive factors for postoperative outcomes $^{12,27,38}$; however, the ACS NSQIP is designed to protect against such analyses to encourage participation of all hospitals.

In summary, we present the largest multicentered cohort of UC patients who had an IPAA procedure stratified by timing of pouch creation. Our data suggest that for UC patients who had a colectomy, delayed pouch creation had a lower rate of postoperative adverse events compared with pouch creation at the time of colectomy. These data are valuable for preoperative risk assessment and counseling of UC patients before IPAA creation. These data can also be used to guide postoperative management and discharge planning for UC patients who underwent an IPAA procedure. Given the rising prevalence of UC, high-quality research, including disease-specific factors and IBD-related medications, is required to delineate the risks and benefits of the surgical management of the disease. Furthermore, better data are needed to elucidate the long-term sequelae after multistaged IPAA creation.

\section{REFERENCES}

1. Kappelman MD, Moore KR, Allen JK, et al. Recent trends in the prevalence of Crohn's disease and ulcerative colitis in a commercially insured US population. Dig Dis Sci. 2013;58:519-25.

2. Shivashankar R, Tremaine WJ, Harmsen WS, et al. Incidence and prevalence of Crohn's disease and ulcerative colitis in Olmsted County, Minnesota from 1970 through 2010. Clin Gastroenterol Hepatol. 2017;15:857-63.

3. Ross H, Steele SR, Varma M, et al; Standards Practice Task Force of the American Society of Colon and Rectal Surgeons. Practice parameters for the surgical treatment of ulcerative colitis. Dis Colon Rectum. 2014;57:5-22.

4. Parks AG, Nicholls RJ. Proctocolectomy without ileostomy for ulcerative colitis. Br Med J. 1978;2:85-8.

5. Magro F, Gionchetti P, Eliakim R, et al; European Crohn's and Colitis Organisation. Third European evidence-based consensus on diagnosis and management of ulcerative colitis. Part 1: definitions, diagnosis, extra-intestinal manifestations, pregnancy, cancer surveillance, surgery, and ileo-anal pouch disorders. J Crohns Colitis. 2017;11:649-70.

6. Geltzeiler CB, Lu KC, Diggs BS, et al. Initial surgical management of ulcerative colitis in the biologic era. Dis Colon Rectum. 2014;57:1358-63.

7. Reich KM, Chang HJ, Rezaie A, et al. The incidence rate of colectomy for medically refractory ulcerative colitis has declined in parallel with increasing anti-TNF use: a time-trend study. Aliment Pharmacol Ther. 2014;40:629-38.

8. Remzi FH, Fazio VW, Gorgun E, et al. The outcome after restorative proctocolectomy with or without defunctioning ileostomy. Dis Colon Rectum. 2006;49:470-7.

9. Weston-Petrides GK, Lovegrove RE, Tilney HS, et al. Comparison of outcomes after restorative proctocolectomy with or without defunctioning ileostomy. Arch Surg. 2008;143:406-12.

10. Samples J, Evans K, Chaumont N, et al. Variant two-stage ileal pouch-anal anastomosis: an innovative and effective alternative to standard resection in ulcerative colitis. J Am Coll Surg. 2017;224:557-63.

11. Swenson BR, Hollenbeak CS, Poritz LS, et al. Modified two-stage ileal pouchanal anastomosis: equivalent outcomes with less resource utilization. Dis Colon Rectum. 2005;48:256-61.

12. Hicks CW, Hodin RA, Bordeianou L. Possible overuse of 3-stage procedures for active ulcerative colitis. JAMA Surg. 2013;148:658-64.

13. Ingraham AM, Richards KE, Hall BL, et al. Quality improvement in surgery: the American College of Surgeons National Surgical Quality Improvement Program approach. Adv Surg. 2010;44:251-67.
14. Khuri SF, Daley J, Henderson W, et al. The Department of Veterans Affairs NSQIP: the first national, validated, outcome-based, risk-adjusted, and peer-controlled program for the measurement and enhancement of the quality of surgical care. National VA Surgical Quality Improvement Program. Ann Surg. 1998;228:491-507.

15. Dindo D, Demartines N, Clavien PA. Classification of surgical complications: a new proposal with evaluation in a cohort of 6336 patients and results of a survey. Ann Surg. 2004;240:205-13.

16. Rencuzogullari A, Stocchi L, Costedio M, et al. Characteristics of learning curve in minimally invasive ileal pouch-anal anastomosis in a single institution. Surg Endosc. 2017;31:1083-92.

17. Fazio VW, Kiran RP, Remzi FH, et al. Ileal pouch anal anastomosis: analysis of outcome and quality of life in 3707 patients. Ann Surg. 2013;257:679-85.

18. Kirat HT, Remzi FH, Kiran RP, et al. Comparison of outcomes after handsewn versus stapled ileal pouch-anal anastomosis in 3109 patients. Surgery. 2009;146:723-9.

19. Ozturk E, Kiran RP, Remzi F, et al. Early readmission after ileoanal pouch surgery. Dis Colon Rectum. 2009;52:1848-53.

20. Farouk R, Pemberton JH, Wolff BG, et al. Functional outcomes after ileal pouchanal anastomosis for chronic ulcerative colitis. Ann Surg. 2000;231:919-26.

21. Meagher AP, Farouk R, Dozois RR, et al. J ileal pouch-anal anastomosis for chronic ulcerative colitis: complications and long-term outcome in 1310 patients Br J Surg. 1998:85:800-3.

22. Hahnloser D, Pemberton JH, Wolff BG, et al. Results at up to 20 years after ilea pouch-anal anastomosis for chronic ulcerative colitis. Br J Surg. 2007;94:333-40.

23. Lightner AL, Mathis KL, Dozois EJ, et al. Results at up to 30 years after ileal pouch-anal anastomosis for chronic ulcerative colitis. Inflamm Bowel Dis. 2017;23:781-90

24. Lovegrove RE, Tilney HS, Heriot AG, et al. A comparison of adverse events and functional outcomes after restorative proctocolectomy for familial adenomatous polyposis and ulcerative colitis. Dis Colon Rectum. 2006;49:1293-306.

25. Lovegrove RE, Constantinides VA, Heriot AG, et al. A comparison of hand-sewn versus stapled ileal pouch anal anastomosis (IPAA) following proctocolectomy: a meta-analysis of 4183 patients. Ann Surg. 2006;244:18-26.

26. Peyrin-Biroulet L, Germain A, Patel AS, et al. Systematic review: outcomes and post-operative complications following colectomy for ulcerative colitis. Aliment Pharmacol Ther. 2016;44:807-16.

27. Sutton JM, Wima K, Wilson GC, et al. Factors associated with 30-day readmission after restorative proctocolectomy with IPAA: a national study. Dis Colon Rectum. 2014:57:1371-8.

28. Seifarth C, Borner L, Siegmund B, et al. Impact of staged surgery on quality of life in refractory ulcerative colitis. Surg Endosc. 2017;31(2):643-649.

29. Mège D, Figueiredo MN, Manceau G, et al. Three-stage laparoscopic ilea pouch-anal anastomosis is the best approach for high-risk patients with inflammatory bowel disease: an analysis of 185 consecutive patients. J Crohns Colitis. 2016;10:898-904.

30. Zittan E, Wong-Chong N, Ma GW, et al. Modified two-stage ileal pouch-ana anastomosis results in lower rate of anastomotic leak compared with traditional two-stage surgery for ulcerative colitis. J Crohns Colitis. 2016;10:766-72.

31. de Silva S, Ma C, Proulx MC, et al. Postoperative complications and mortality following colectomy for ulcerative colitis. Clin Gastroenterol Hepatol. 2011;9:972-80.

32. Kulaylat AS, Kulaylat AN, Schaefer EW, et al. Association of preoperative anti-tumor necrosis factor therapy with adverse postoperative outcomes in patients undergoing abdominal surgery for ulcerative colitis. JAMA Surg. 2017;152:e171538.

33. Wilson MZ, Connelly TM, Tinsley A, et al. Ulcerative colitis is associated with an increased risk of venous thromboembolism in the postoperative period: the results of a matched cohort analysis. Ann Surg. 2015;261:1160-6.

34. Bollegala N, Jackson TD, Nguyen GC. Increased postoperative mortality and complications among elderly patients with inflammatory bowel diseases: an analysis of the National Surgical Quality Improvement Program cohort. Clin Gastroenterol Hepatol. 2016;14:1274-81.

35. Pedersen M, Cromwell J, Nau P. Sarcopenia is a predictor of surgical morbidity in inflammatory bowel disease. Inflamm Bowel Dis. 2017;23:1867-72.

36. Merrill A, Millham F. Increased risk of postoperative deep vein thrombosis and pulmonary embolism in patients with inflammatory bowel disease: a study of National Surgical Quality Improvement Program patients. Arch Surg. 2012;147:120-4

37. Gunnells DJ Jr, Morris MS, DeRussy A, et al. Racial disparities in readmissions for patients with inflammatory bowel disease (IBD) after colorectal surgery. $J$ Gastrointest Surg. 2016;20:985-93.

38. Parc Y, Reboul-Marty J, Lefevre JH, et al. Restorative proctocolectomy and ileal pouch-anal anastomosis. Ann Surg. 2015;262:849-53. 